\title{
Distributions of Time- and Distance-Headways in the Nagel-Schreckenberg Model of Vehicular Traffic: Effects of Hindrances
}

\author{
Debashish Chowdhury ${ }^{1}$, Abhay Pasupathy and Shishir Sinha \\ Physics Department, Indian Institute of Technology \\ Kanpur 208016, India
}

\begin{abstract}
In the Nagel-Schreckenberg model of vehicular traffic on single-lane highways vehicles are modelled as particles which hop forward from one site to another on a one dimensional lattice and the inter-particle interactions mimic the manner in which the real vehicles influence each other's motion. In this model the number of empty lattice sites in front of a particle is taken to be a measure of the corresponding distance-headway $(\mathbf{D H})$. The time-headway $(\mathbf{T H})$ is defined as the time interval between the departures (or arrivals) of two successive particles recorded by a detector placed at a fixed position on the model highway. We investigate the effects of spatial inhomogeneities of the highway (static hindrances) on the $\mathrm{DH}$ and TH distributions in the steady-state of this model.
\end{abstract}

PACS. 05.40. $+\mathrm{j}$ - Fluctuation phenomena, random processes, and Brownian motion.

PACS. 05.60. $+\mathrm{w}$ - Transport processes: theory.

PACS. 89.40.+k - Transportation.

${ }^{1}$ To whom all correspondence should be addressed; E-mail: debch@iitk.ernet.in 


\section{Introduction:}

The continuum models of traffic flow [1-3] are analogues of the "hydrodynamic" models of fluid flow while the kinetic theories of vehicular traffic [4-6], which are extensions of the kinetic theory of gases, and the car-following models [7-10] as well as the discrete "particle-hopping" models [11-17] are analogues of the "microscopic" models of interacting particles commonly studied in statistical mechanics. In this paper we focus our attention on a specific particle-hopping model, namely, the Nagel-Schreckenberg (NS) model [11] of vehicular traffic on idealized single-lane highways; this model may be regarded as a model of interacting particles driven far from equilibrium and the dynamical phenomena exhibited by this model of traffic may be treated as problems of non-equilibrium statistical mechanics.

The distance-headway(DH) is defined as the distance from a selected point on the lead vehicle $(\mathbf{L V})$ to the same point on the following vehicle $(\mathbf{F V})$. Usually, the front edges or bumpers are selected [18]. The time-headway(TH) is defined as the time interval between the departures (or arrivals) of two successive vehicles recorded by a detector placed at a fixed position on the highway [18]. The DH and TH are not merely of academic interest to statistical physicists, but are also of practical interest in traffic engineering where these two distributions are regarded as important characteristic of traffic flow $[18,19]$. For example, larger headways provide greater margins of safety whereas higher capacities of the highway require smaller headways. 
Recent investigations by several groups of statistical physicists [20-24] have helped in gaining insight into the nature of the "jamming transition" (i.e., the transition from the "free-flowing" dynamical phase to the "jammed" dynamical phase) in the NS model. On the other hand, simultaneously, NS model is being generalized and extended [25-37] to capture more and more details of vehicular traffic flow so that the generalized/extended model may, ultimately, find practical use in traffic engineering [38-39]. Extending our earlier numerical works on the DH distribution in the steady-state of the NS model, we point out here interesting similarities as well as crucial differences between the "jamming transition" and the gas-liquid phase transition in a simple fluid in equilibrium. Moreover, in this paper we also present a simple analytical calculation of the TH distribution in the steady-state of a special case of the NS model using an interesting quantity introduced in ref.[15]. Furthermore, we study the effects of spatial inhomogeneities of the highway (static hindrances) on the $\mathrm{DH}$ and $\mathrm{TH}$ distributions in the steady-state of the NS model.

The NS model and some of its most important features are summarized in section 2. We report numerical results on the $\mathrm{DH}$ distribution in the steadystate of the NS model, in the absence as well as in the presence of hindrances, in section 3 where we also draw attention to the analogies and differences between the jamming transition in the NS model and the gas-liquid transition. Our calculations of the TH distribution in the steady-state of the NS model are given in the section 4 , where we also study the effects of hindrances on this 
distribution. Finally, we summarize our main results and conclusions in section 5 .

\section{The Models:}

In the NS model a lane is represented by a one-dimensional lattice of $L$ sites. Each of the lattice sites can be either empty or occupied by at most one "vehicle". If periodic boundary condition is imposed, the density $c$ of the vehicles is $N / L$ where $N(\leq L)$ is the total number of vehicles. In the NS model [11] the speed $V$ of each vehicle can take one of the $V_{\max }+1$ allowed integer values $V=0,1, \ldots, V_{\max }$. Suppose, $V_{n}$ is the speed of the $n$-th vehicle at time $t$. At each discrete time step $t \rightarrow t+1$, the arrangement of $N$ vehicles is updated in parallel according to the following "rules":

Step 1: Acceleration. If, $V_{n}<V_{\max }$, the speed of the $n$-th vehicle is increased by one, i.e., $V_{n} \rightarrow V_{n}+1$.

Step 2: Deceleration (due to other vehicles). If $d_{n}$ is the gap in between the $n$-th vehicle and the vehicle in front of it, and if $d_{n} \leq V_{n}$, the speed of the $n$-th vehicle is reduced to $d_{n}-1$, i.e., $V_{n} \rightarrow d_{n}-1$.

Step 3: Randomization. If $V_{n}>0$, the speed of the $n$-th vehicle is decreased randomly by unity (i.e., $\left.V_{n} \rightarrow V_{n}-1\right)$ with probability $p(0 \leq p \leq 1)$; $p$, the random deceleration probability, is identical for all the vehicles and does not change during the updating. 
Step 4: Vehicle movement. Each vehicle is moved forward so that $X_{n} \rightarrow X_{n}+V_{n}$ where $X_{n}$ denotes the position of the $n$-th vehicle at time $t$.

The specific update rule of the NS model requires a nonvanishing braking probability $p$ for the model to yield a realistic description of traffic flow [12] and, thus, the NS model may be regarded as stochastic cellular automata [40]. Effectively free flow of traffic takes place when the density of vehicles is sufficiently low whereas high density leads to congestion and traffic jams. One of our aims is to point out some similarities as well as differences between the "jamming transition" in the NS model [20-24], which is a non-equilibrium driven system, and the gas-liquid phase transition in a fluid in equilibrium.

The asymmetric simple exclusion process (ASEP) is one of the simplest models of driven systems of interacting particles; it is often treated as a carricature of traffic flow. The relations between this model and the $V_{\max }=1$ limit of the NS model has been elucidated in the literature [24]. Some dramatic effects of quenched disorder (static hindrances) on the steady state of this model have been investigated $[41,42]$. Our main aim in this paper is to investigate the effects of static hindrances on the DH and TH distributions in the NS model.

Emmerich and Rank [32] introduced an extra step of update rule before all the other steps of updating in the NS model to mimic the effects of static traffic hindrance: a segment of length $L_{\text {hind }}$ on the highway is identified as the hindrance and the speed of all the vehicles found within that segment of the highway are reduced to half of their current speed. A more general model of 
traffic flow in the presence of "quenched disorder" in the highway was formulated by Csahok and Vicsek [33] in terms of an "inverse permeability" associated with each site. We shall use the simpler rule introduced by Emmerich and Rank [32].

For the convenience of our analytical calculations, following Schreckenberg et al.[14], we assume the sequence of steps $2-3-4-1$, instead of $1-2-3-4$; the advantage is that there is no vehicle with $V=0$ immediately after the acceleration step. Consequently, if $V_{\max }=1$, we can then use a binary site variable $\sigma$ to describe the state of each site; $\sigma=0$ represents an empty site and $\sigma=1$ represents a site occupied by a vehicle whose speed is unity.

\section{Distance-Headway Distributions and the Na- ture of the Jamming Transition}

Using the sequence of steps $2-3-4-1$, as explained earlier, an $n$-cluster configuration in the steady-state of the NS model is represented by $\left(\sigma_{1}, \sigma_{2}, \ldots, \sigma_{n}\right)$. The number of empty lattice sites, $n$, in front of a vehicle is taken to be a measure of the corresponding DH. Within the 2-cluster approximation, the DH distribution, $\mathcal{P}_{2 c}^{d h}(n)$, in the steady-state of the NS model with $V_{\max }=1$ has been calculated analytically following the methods of the site-oriented meanfield (SOMF) theory [14]. In this approximation,

$$
\mathcal{P}_{2 c}^{d h}(0)=\mathcal{C}(\underline{1} \mid 1)
$$


and

$$
\mathcal{P}_{2 c}^{d h}(n)=\mathcal{C}(\underline{1} \mid 0)\{\mathcal{C}(\underline{0} \mid 0)\}^{n-1} \mathcal{C}(\underline{0} \mid 1), \quad \text { for } \quad n \geq 1
$$

where, $\mathcal{C}$ gives the 2 -cluster steady-state configurational probability for the argument configuration and the underlined imply the conditional, as usual. The expressions for the various $\mathcal{C}$ s are given by $[14,24]$

$$
\begin{aligned}
\mathcal{C}(\underline{0} \mid 0) & =\mathcal{C}(0 \mid \underline{0})=1-\frac{y}{d} \\
\mathcal{C}(\underline{1} \mid 0) & =\mathcal{C}(0 \mid \underline{1})=\frac{y}{c} \\
\mathcal{C}(\underline{0} \mid 1) & =\mathcal{C}(1 \mid \underline{0})=\frac{y}{d} \\
\mathcal{C}(\underline{1} \mid 1) & =\mathcal{C}(1 \mid \underline{1})=1-\frac{y}{c}
\end{aligned}
$$

where

$$
y=\frac{1}{2 q}(1-\sqrt{1-4 q c d}),
$$

$q=1-p$ and $d=1-c$. It turned out that the 2 -cluster approxmation is exact for $V_{\max }=1$ [14]. Therefore, in terms of $c$ and $p$, the exact DH distribution in the steady-state of the NS model with $V_{\max }=1$ is given by

$$
\mathcal{P}^{d h}(0)=1-(y / c)
$$

and

$$
\mathcal{P}^{d h}(n)=\left\{y^{2} /(c d)\right\}[1-(y / d)]^{n-1} \quad \text { for } \quad n \geq 1
$$

where, for the given $c$ and $p, y$ can be obtained from equation (7). The same exact DH distribution (8-9) has also been derived independently [15] within the 
framework of car-oriented mean-field (COMF) theory. The DH distributions in the NS model for all $V_{\max }>1$ have been computed numerically by carrying out computer simulation [24].

The DH distribution in the steady-state of the NS model simultaneously exhibits two peaks over an intermediate regime of the vehicle densities, provided $V_{\max }>1$ and $p$ is sufficiently large. In contrast, when $V_{\max }=1$, no such twopeak structure is exhibited at any density, for any $p$, by the DH distribution which is given by the exact expression (8-9). We have now estimated, as functions of $p$, the densities, $c_{s}$ and $c_{\ell}$, corresponding to the smallest and the largest densities where two-peak structure is exhibited by the $\mathrm{DH}$ distribution in the NS model $\left(2 \leq V_{\max } \leq 5\right) . c_{s}$ and $c_{\ell}$ are plotted against $1-p$ for $V_{\max }=2 \mathrm{in}$ fig.1.

The occurrence of the two-peak structure for $V_{\max }>1$ has been interpreted $[24,43]$ as a signature of "two-phase coexistence"; the two coexisting phases, namely, the "free-flowing phase" and the "jammed phase" being the analogues of the gas phase and the liquid phase, respectively, of a simple fluid in equilibrium. In this scenario, for a given $p$ (and given $V_{\max }$ ), it is tempting to identify $c_{s}$ as the analogue of the density corresponding to the onset of two-phase coexistence in a fluid while $c_{\ell}$ would be the corresponding largest density up to which these two phases continue to coexist at a given temperature; accordingly, the regime of density in between these two curves would be the analogue of the two-phase coexistence region for a fluid and the uppermost tip of this region could be 
interpreted as an analogue of the critical point. Further, this analogy would suggest that $1-p$ is the analogue of temperature; increase of $p$ (i.e., decrease of $1-p$ ) drives the system towards "condensation".

In spite of this apparent analogy, there are crucial differences between the "jamming transition" and the gas-liquid transition in a simple fluid. First of all, the fluid is in equilibrium whereas the model vehicular traffic under consideration is in a non-equlibrium steady-state. More important difference is that the size of the jams remain finite even in the limit of infinite system size [24] and, therefore, cannot be identified as a true dynamical phase.

When the DH distribution exhibits two peaks simultaneously, the peak at vanishing $\mathrm{DH}$ reflects the fact that the total number of vehicles held up simultaneously in the various jams on this model highway is a finite fraction of all the vehicles on this highway; these jams arise from spontaneous fluctuations in the driven system of interacting particles and, therefore, can appear anywhere in the system. In contrast, a hindrance can induce recurring jams at (and near) its own location, as demonstrated in fig.2, because of the bottlenecks against traffic flow created by it. The DH distributions for 5 different values of $L_{\text {hind }}$ are plotted in fig.3, all for the same density $c=0.05$ and for the same $p=0.5$. In this figure the absence of two-peak structure for $L_{\text {hind }}=0$ indicates that, for $p=0.5$, the density $c=0.05$ is not high enough to have a finite fraction of the vehicles, simultaneously, held up in various jams created by spontaneous fluctuations. However, for the same set of values of $c$ and $p$, two-peak structure 
occurs in the $\mathrm{DH}$ distribution with the increase of $L_{\text {hind }}$; this is a consequence of jams caused by the bottleneck effect of the hindrance.

\section{Time-Headway Distribution:}

There are several earlier papers, published by statisticians and traffic engineers, where the form of the TH distribution has been derived on the basis of heuristic arguments [44]. In this section we begin by presenting a simple derivation of this distribution for $V_{\max }=1$ using a quantity introduced in ref.[15] in the context of the COMF theory. However, we could compute the corresponding TH distributions for $V_{\max }>1$ [45] and the effects of hindrances on these distributions only through computer simulation.

We label the position of the detector by $j=0$, the site immediately in front of it by $j=1$, and so on. The detector clock resets to $t=0$ everytime a vehicle leaves the detector site. We begin our analytical calculations for $V_{\max }=1$ by writing $\mathcal{P}^{t h}(t)$, the probability of a time headway $t$ between a LV and the FV,

as

$$
\mathcal{P}^{t h}(t)=\sum_{t_{1}=1}^{t-1} P\left(t_{1}\right) Q^{\prime}\left(t-t_{1} \mid t_{1}\right)
$$

where $P\left(t_{1}\right)$ is the probability that there is a time interval $t_{1}$ between the departure of the $\mathrm{LV}$ and the arrival of the FV at the detector site and $Q^{\prime}\left(t-t_{1} \mid t_{1}\right)$ is the conditional probability that the $\mathrm{FV}$ halts for $t-t_{1}$ time steps when it arrived at the detector site $t_{1}$ time steps after the departure of the LV. 
Suppose, $g(t)$ is the probability that a vehicle moves in the next (i.e., in the $t+1$-th time step). Therefore, $\bar{g}(t)=1-g(t)$ is the probability that a vehicle does not move in the next time step. It has been shown [15] that $g=q\left[1-\mathcal{P}^{d h}(0)\right]$. Using equation (8) for $\mathcal{P}^{d h}(0)$ we get

$$
g=q y / c
$$

and, hence, $\bar{g}=1-q y / c$, where $y$ is given by the equation (7). Moreover, since $y$ satisfies the equation $q y^{2}-y+c d=0$, it follows that

$$
(1-q y / c)(1-q y / d)=p
$$

In order to calculate $P\left(t_{1}\right)$ we need to consider all those spatial configurations at $t=0$ from which the FV can reach the detector site within $t_{1}$ steps. For all configurations with $t_{1}>n, t_{1}-1$ time steps elapse in crossing $n-1$ linkss (as the last link is crossed certainly at the last time step). Thus

$$
P\left(t_{1}\right)=\sum_{n=1}^{t_{1}} \Pi(n) q^{n} p^{t_{1}-n} \quad{ }^{t_{1}-1} C_{n-1}
$$

where

$$
\Pi(n)=\mathcal{C}(1 \mid \underline{0})\{\mathcal{C}(0 \mid \underline{0})\}^{n-1}
$$

and, hence, we get

$$
P\left(t_{1}\right)=\mathcal{C}(1 \mid \underline{0}) q[\mathcal{C}(0 \mid \underline{0}) q+p]^{t_{1}-1}=\frac{q y}{d}\left(1-\frac{q y}{d}\right)^{t_{1}-1}
$$

which is the exact analytical expression for $P\left(t_{1}\right)$.

Next, we calculate $Q\left(t-t_{1} \mid t_{1}\right)$ by expressing it in terms of $g$ and $\bar{g}$. When the FV arrives at the detector site exactly $t_{1}$ time steps after the departure of 
the LV, the LV can be at any of the sites labeled by $1, \cdots, t_{1}+1$. The probability that the LV stays at the site ' 1 ' is $\bar{g}^{t_{1}}$ and, therefore, the probability that the $\mathrm{LV}$ is not at the site ' 1 ' is $1-\bar{g}^{t_{1}}$.

If the LV is not at site ' 1 ' then the probability that the FV halts at the detector site for exactly $t-t_{1}$ time steps is $p^{t-t_{1}-1} q$ because it should stop due to randomisation for exactly $t-t_{1}-1$ steps and move at the last step. Hence, the contribution to $Q^{\prime}\left(t-t_{1} \mid t_{1}\right)$ when the $L V$ is not at site ' 1 ' is

$$
\left(1-\bar{g}^{t_{1}}\right) p^{t-t_{1}-1} q
$$

On the other hand, when the LV is at site ' 1 ' then it will have to move so that the FV is able to leave the detector site after $t-t_{1}$ steps. Suppose the LV moves from ' 1 ' after $k$ steps ( $k$ varies from 1 to $\left.t-t_{1}-1\right)$. Then the FV will have to stay at the detector site for next $t-t_{1}-1-k$ steps due to randomisation and move in the last step. Hence, the contribution to $Q^{\prime}\left(t-t_{1} \mid t_{1}\right)$ when LV is at site ' 1 ' is

$$
\bar{g}^{t_{1}} g q\left(\sum_{k=1}^{t-t_{1}-1} \bar{g}^{k-1} p^{t-t_{1}-1-k}\right)=\bar{g}^{t_{1}} g q \frac{\left[(\bar{g})^{t-t_{1}-1}-(p)^{t-t_{1}-1}\right]}{\bar{g}-p}
$$

Therefore, combining (I) and (II) we get

$$
Q^{\prime}\left(t-t_{1} \mid t_{1}\right)=\left(1-\bar{g}^{t_{1}}\right) p^{t-t_{1}-1} q+\bar{g}^{t_{1}} g q \frac{\left[(\bar{g})^{t-t_{1}-1}-(p)^{t-t_{1}-1}\right]}{\bar{g}-p}
$$

Finally, substituting the exact expressions (15) and (16) into (10) we get

$$
\begin{aligned}
\mathcal{P}^{t h}(t)=\left[\frac{q y}{c-y}\right]\{1 & -(q y / c)\}^{t-1}+\left[\frac{q y}{d-y}\right]\{1-(q y / d)\}^{t-1} \\
& -\left[\frac{q y}{c-y}+\frac{q y}{d-y}\right] p^{t-1}-q^{2}(t-1) p^{t-2}
\end{aligned}
$$


where, for the given $c$ and $p, y$ can be obtained from equation (7). The invariance of the distribution (17) under the interchange of $c$ and $1-c$, is a manifestations of the well-known "particle-hole" symmetry in the problem which breaks down for all $V_{\max }>1[46]$.

The flux $q$ of the vehicles can be written as $q=N / T$ where $T=\sum_{i=1}^{N} t_{i}$ is the sum of the time headways recorded for all the $N$ vehicles. Therefore, one can rewrite $q$ as $q=1 / T_{a v}$ where $T_{a v}=(1 / N) \sum_{i} t_{i}$ is the average TH. Therefore, $T_{a v}$ is expected to exhibit a minimum at $c=c_{m}$ with the variation of density $c$ of the vehicles. We observed that the trend of variation of the most-probable TH, $T_{m p}$, with $c$ is similar to that of $T_{a v}$ with $c$ [45]. Moreover, because of the particle-hole symmetry, the $T_{m p}$ versus $c$ curve is symmetric about $c=1 / 2$ in the NS model with $V_{\max }=1$. But this symmetry is lost when $V_{\max }>1$. The fact that $T_{m p}$ (and $T_{a v}$ ) versus $c$ curve exhibits a minimum is consistent with one's intuitive expectation that both at very low and very high densities there are long time gaps in between the departures of two successive vehicles from a given site.

Two typical sets of curves showing the effects of the hindrances on the TH distribution in the steady-state of the NS model are shown in fig.4. At low density of the vehicles, increase of the length of the hindrance leads to significant broadening of the distribution although its effect on the magnitute of the most probable TH is weak (see fig.4a). On the other hand, at moderate and high densities, the broadening of the TH distribution caused by increase of $L_{\text {hind }}$ is 
much weaker (see fig.4b).

\section{Summary and Conclusion:}

In this paper we have investigated two important characteristics of traffic flow on highways, namely, DH and TH distributions, starting from models that incorporate explicitly vehicle-vehicle and road-vehicle interactions. We have compared and contrasted the effects of static hindrance on the highway and those of the LV, which may be viewed as a dynamic hindrance, against the forward movement of the FV. In the absence of any static hindrance jams can appear anywhere in the system because of spontaneous fluctuations and the dynamic hindrance caused by a slower LV on the FV. On the other hand, in the presence of a static hindrance, jams take place at (and near) the hindrance, in addition to those formed by spontaneous fluctuations, because of bottleneck it creates against the traffic flow.

We have extracted some informations on the "structures" in the spatial oraganization of the vehicles on the highway from the DH distribution. We have demonstrated the effects of the bottleneck against traffic flow created by static hindrances on the $\mathrm{DH}$ distribution thereby elucidating the physical meaning of the two-peak structures observed earlier in the $\mathrm{DH}$ distribution over some intermediate regime of density when $V_{\max }>1$ and $p$ is sufficiently large.

The TH distributions in the steady state of the NS model and the trend 
of their variation with density are in good qualitative agreement with the corresponding empirical data [18]. Our results demonstrate that, in spite of its simplicity, the NS model captures the essential qualitative features of the TH distribution of vehicular traffic on highways. Besides, the extent of broadening of the TH distribution caused by a hindrance depends on the density of the vehicles; the higher is the density the larger is the broadening. Hoever, at all densities, the effect of the hindrance on the magnitude of the $T_{m p}$ is very weak.

In this paper we have considered only the original version of the NS model for vehicular traffic on single-lane highways [11] and an extended version that includes a specific type of inhomogeneities of the highway (namely, static hindrances) [32]. We have not attempted any direct quantitative comparison of our results with the corresponding empirical data from highway traffic because, we believe, such comparisons will be possible only after several of the realistic generalizations and extensions [25-37], proposed recently in the literature, are incorporated in the model. Results of our ongoing works in this direction will be published elsewhere [47].

Acknolwdegements: We dedicate this paper to Professor Zittartz on the occassion of his 60th birthday. The research career of the senior author (DC) has been influenced strongly by the generous encouragements received from Professor Zittartz over the last 14 years. This paper is in an area where present Members of Professor Zittartz's group as well as his former students, postdocs and assistants have made most of the influential contributions. DC also thanks 
D. Stauffer, A. Schadschneider, L. Santen and anonymous referees for useful comments and the Alexander von Humboldt Foundation, for partial support through a research equipment grant. 


\section{References}

[1] M.J. Lighthill and G.B. Whitham, Proc. Roy. Soc. Lond. A 229, 281 (1955)

[2] B.S. Kerner, S.L. Klenov and P. Konhaeuser, Phys. Rev. E 56, 4200 (1997)

[3] M.Y. Choi and H.Y. Lee, Phys. Rev. E 52, 5979 (1995)

[4] I. Prigogine and R. Herman, Kinetic Theory of Vehicular Traffic (Elsevier, Amsterdam, 1971)

[5] D. Helbing, Physica A 219, 375 (1995); 219, 391 (1995); 233, 253 (1995)

[6] T. Nagatani, J. Phys. Soc. Jap. 66, 1219 (1997)

[7] see, for reviews, R. Herman and K. Gardels, Sci.Amer.209,(6), 35 (1963); D.C. Gazis, Science, 157, 273 (1967)

[8] M. Bando, K. Hasebe, A. Nakayama, A. Shibata and Y. Sugiyama, Phys. Rev. E 51, 1035 (1995)

[9] S. Yukawa and M. Kikuchi, J. Phys. Soc. Jap. 64, 35 (1995); 65, 916 (1996).

[10] T. Nagatani, J. Phys. Soc. Jap. 65, 2333 (1996).

[11] K. Nagel and M. Schreckenberg, J. Physique I, 2, 2221 (1992).

[12] K. Nagel and H.J. Herrmann, Physica A 199, 254 (1993)

[13] A. Schadschneider and M. Schreckenberg, J. Phys. A 26, L679 (1993) 
[14] M. Schreckenberg, A. Schadschneider, K. Nagel and N. Ito, Phys.Rev.E, 51, 2939 (1995).

[15] A. Schadschneider and M. Schreckenberg, J. Phys. A 30, L69 (1997)

[16] A. Schadschneider and M. Schreckenberg, J. Phys. A 31, (1998)

[17] D.E. Wolf, M. Schreckenberg and A. Bachem (eds.) Traffic and Granular Flow (World Scientific, 1996); Proc. of the Conference "Traffic and Granular Flow '97', Duisburg, Oct.5-8, 1997 (to appear).

[18] A.D. May, Traffic Flow Fundamentals (Prentice-Hall, 1990)

[19] W. Leutzbach, Introduction to the Theory of Traffic Flow (Springer, Berlin, 1988)

[20] L.C.Q. Vilar and A.M.C. De souza, Physica A 211, 84 (1994)

[21] G. Csanyi and J. Kertesz, J. Phys. A 28, L427 (1995); M. Sasvari and J. Kertesz, Phys. Rev.E 56, 4104 (1997).

[22] S. Luebeck, M. Schreckenberg and K.D. Usadel, Phys. Rev. E 57, 1171 (1997); S. Luebeck, L. Roters and K.D. Usadel, Duisburg Preprint.

[23] B. Eisenblaetter, L. Santen, A. Schadschneider and M. Schreckenberg, Phys. Rev. E 57, 1309 (1998).

[24] D. Chowdhury, A. Majumdar, K. Ghosh, S. Sinha and R.B. Stinchcombe, Physica A 246, 471 (1997). 
[25] K. Nagel and M. Paczuski, Phys. Rev. E 51, 2909 (1995)

[26] N. Boccara, H. Fuks and Q. Zeng, J. Phys. A 30, 3329 (1997)

[27] H. Emmerich and E. Rank, Physica A 234, 676 (1997)

[28] S.C. Benjamin, N.F. Johnson and P.M. Hui, J. Phys. A 29, 3119 (1996)

[29] M. Takayasu and H. Takayasu, Fractals, 1, 860 (1993)

[30] A. Schadschneider and M. Schreckenberg, Ann. der Phys. 6, 541 (1997).

[31] S. Migowsy, T. Wanschura and P. Rujan, Z. Phys. B 95, 407 (1994)

[32] H. Emmerich and E. Rank, Physica A 216, 435 (1995)

[33] Z. Csahok and T. Vicsek, J. Phys. A 27, L591 (1994)

[34] D. Ktitarev, D. Chowdhury and D.E. Wolf, J. Phys. A 30, L221 (1997)

[35] M. Rickert, K. Nagel, M. Schreckenberg and A. Latour, Physica A 231, $534(1996)$

[36] P. Wagner, K. Nagel and D.E. Wolf, Physica A 234, 687 (1997)

[37] D. Chowdhury, D. E. Wolf and M. Schreckenberg, Physica A 235, 417 (1997)

[38] M. Rickert and K. Nagel, Int. J. Mod. Phys.C 8, (1997)

[39] J. Esser and M. Schreckenberg, Int. J. Mod.Phys.C 8, 1025 (1997). 
[40] S. Wolfram, Theory and Applications of Cellular Automata, (World Scientific, 1986).

[41] S.A. Janowsky and J.L. Lebowitz, Phys. Rev. A 45, 618 (1992); G. Schütz, J. Stat. Phys. 75, 471 (1993).

[42] G. Tripathy and M. Barma, Phys. Rev. Lett. 78, 3039 (1997).

[43] S. Krauss, P. Wagner and C. Gawron, Phys. Rev. E 54, 3707 (1996) and Phys. Rev. E 55, 5597 (1997).

[44] See chapter 2 of ref.[1] and references therein.

[45] K. Ghosh, A. Majumdar and D. Chowdhury, Phys. Rev. E (in press).

[46] A. Schadschneider, in: Proc. of the Conference "Traffic and Granular Flow '97', Duisburg, Oct.5-8, 1997 (to appear).

[47] D. Chowdhury, S. Sinha and A. Pasupathy (to be published). 


\section{Figure Captions:}

Fig.1: $c_{\ell}$ and $c_{s}$ in the NS model are plotted against $1-p$ when $V_{\max }=2$ (see the text for the definitions of $c_{\ell}$ and $c_{s}$ ).

Fig.2: The "space-time diagram" showing the time-evolution of the traffic in the NS model $\left(V_{\max }=5, p=0.5\right)$ in the presence of ten hindrances, each of length 5, put randomly along the highway of length 1000. Each of the black dots represents a vehicle.

Fig.3: The distance-headway distributions corresponding to the vehicle density $c=0.05$ in the NS model with $V_{\max }=5(p=0.5)$ for five different values of the hindrance length are compared with that in the absence of hindrance. The discrete data points obtained from computer simulation correspond to $L_{\text {hind }}=$ $0(+), L_{\text {hind }}=1(\times), L_{\text {hind }}=2(*), L_{\text {hind }}=3(\square), L_{\text {hind }}=4(\mathbf{\square}), L_{\text {hind }}=5(\circ)$ while the continuous curves are merely guides to the eye.

Fig.4: The time-headway distributions in the NS model $\left(V_{\max }=5\right)$, in the presence of hindrance, for vehicle densities (a) $c=0.1$ and (b) $c=0.5$. The symbols corresponding to $L_{\text {hind }}=0,1,2,3,4$ and 5 are identical to those in fig.3. 


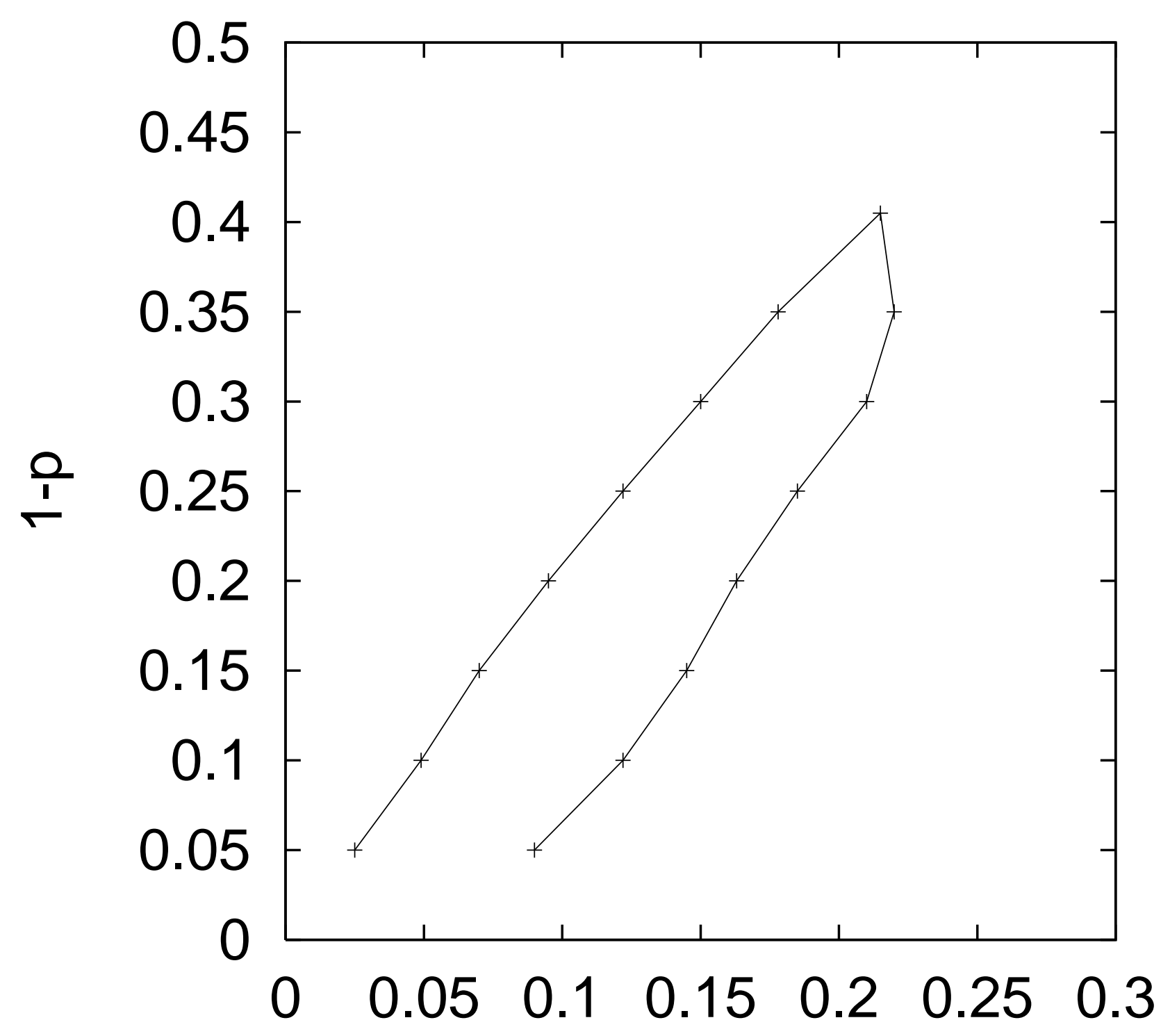

Density 
$\stackrel{\mathbb{E}}{\underline{\underline{F}}}$

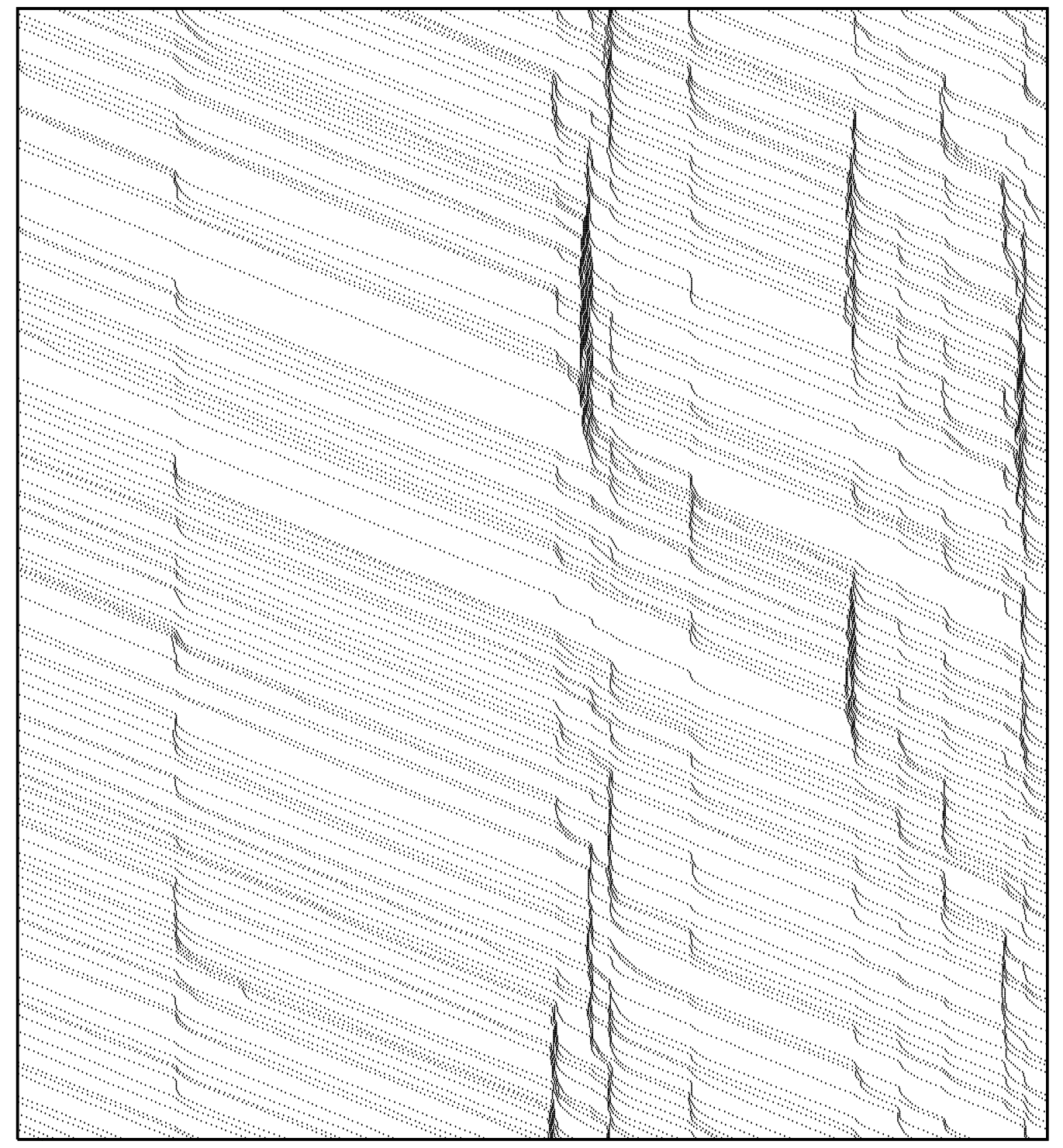

Distance 
Distance headway distribution for $\mathrm{c}=0.05$

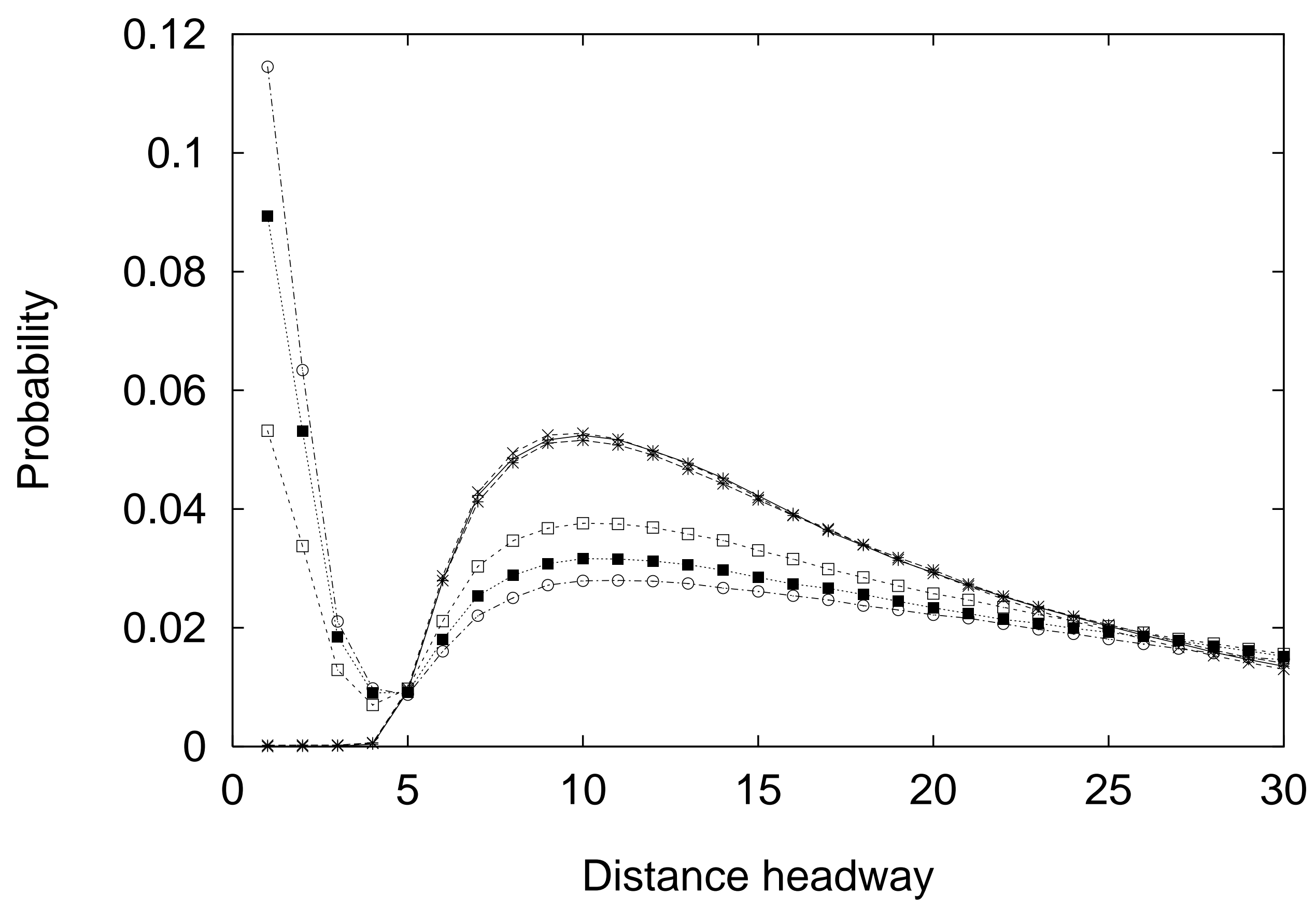


Time headway distribution for $\mathrm{c}=0.10$

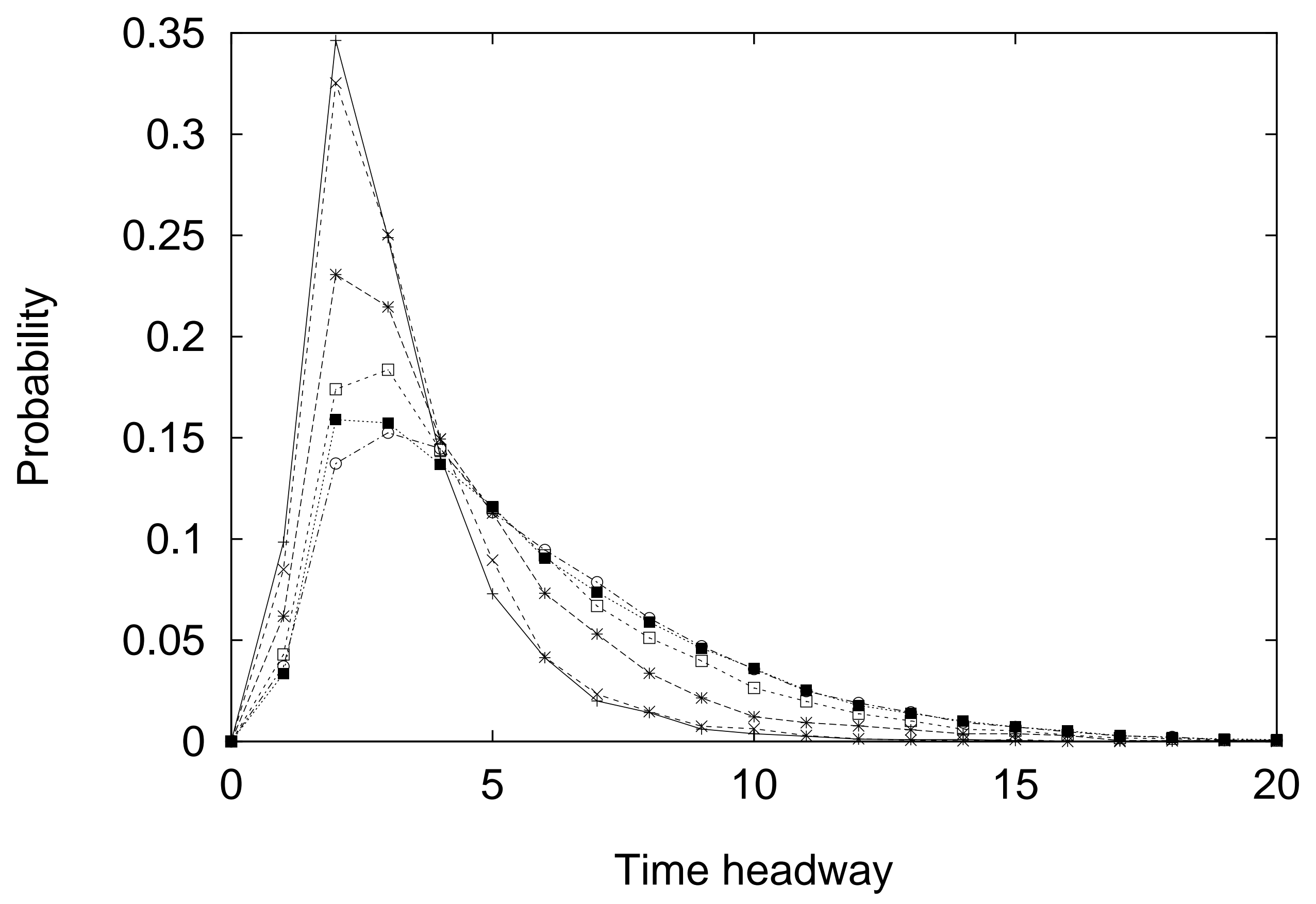


Time headway distribution for $\mathrm{c}=0.50$

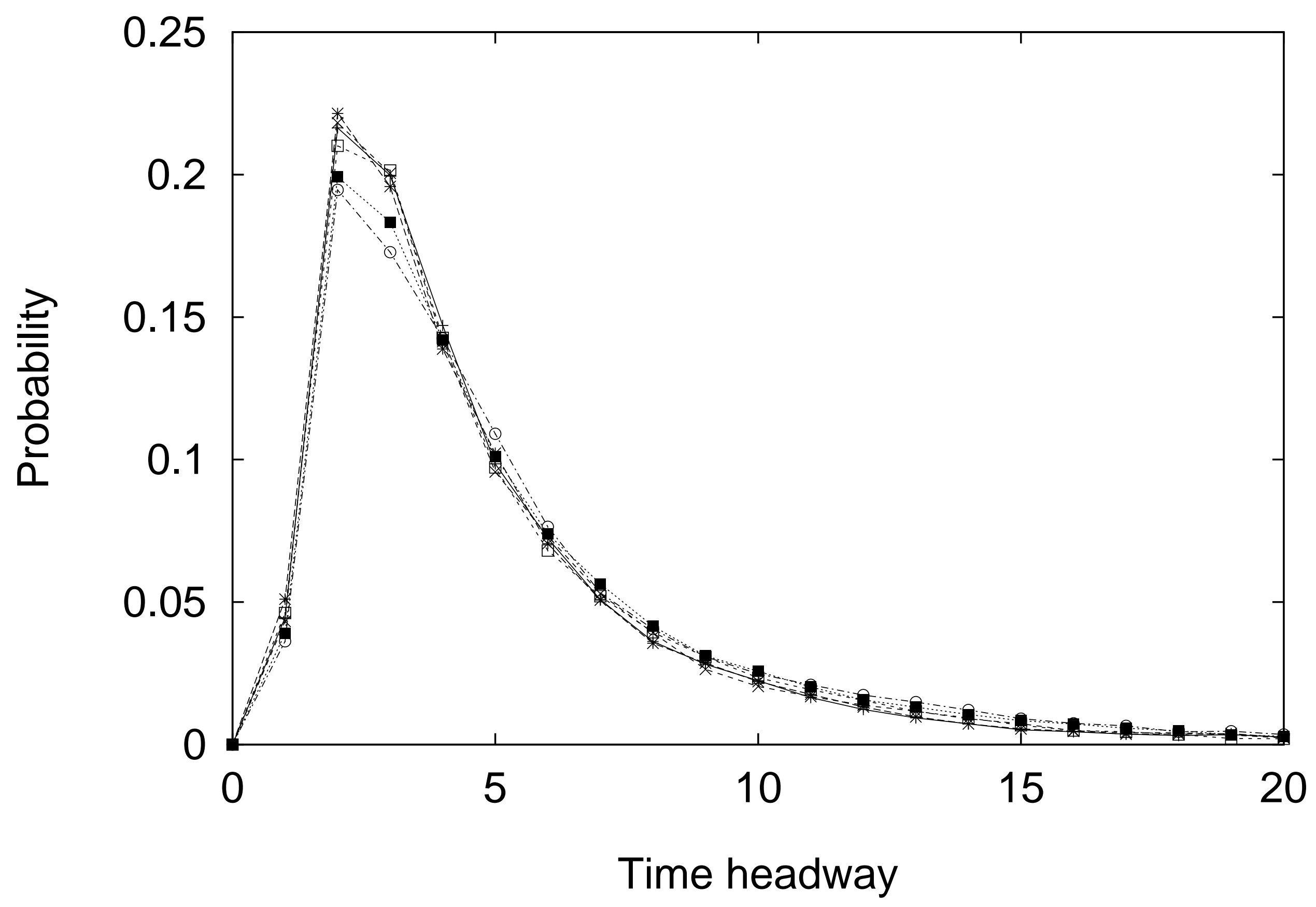

